\title{
A hydrodynamic model for granular material flows including segregation ef- fects
}

\author{
Dominik Gilberg ${ }^{1,2, \star}$, Axel Klar $^{2}$, and Konrad Steiner ${ }^{1}$ \\ ${ }^{1}$ Fraunhofer ITWM, Germany \\ ${ }^{2}$ TU Kaiserslautern, Germany
}

\begin{abstract}
The simulation of granular flows including segregation effects in large industrial processes using particle methods is accurate, but very time-consuming. To overcome the long computation times a macroscopic model is a natural choice. Therefore, we couple a mixture theory based segregation model to a hydrodynamic model of Navier-Stokes-type, describing the flow behavior of the granular material. The granular flow model is a hybrid model derived from kinetic theory and a soil mechanical approach to cover the regime of fast dilute flow, as well as slow dense flow, where the density of the granular material is close to the maximum packing density. Originally, the segregation model has been formulated by Thornton and Gray for idealized avalanches. It is modified and adapted to be in the preferred form for the coupling. In the final coupled model the segregation process depends on the local state of the granular system. On the other hand, the granular system changes as differently mixed regions of the granular material differ i.e. in the packing density. For the modeling process the focus lies on dry granular material flows of two particle types differing only in size but can be easily extended to arbitrary granular mixtures of different particle size and density. To solve the coupled system a finite volume approach is used. To test the model the rotational mixing of small and large particles in a tumbler is simulated.
\end{abstract}

\section{Introduction}

Granular materials are the subject of intensive research for decades already, because they are a part of humans daily lives. Not only in geophysics (dunes and avalanches) or agriculture (corn silos), but especially many different industrial processes deal with granular materials. The nature of the granular particles can strongly influence the behavior of the material and can lead to different phenomena. One of those phenomena is the segregation effect of particles due to different size, density or shape. Especially, in the food and pharmaceutical industry segregation leads to problems, as it counteracts the wanted mixing processes. This can worsen the quality of the products. Therefore, it is helpful to find a model that can describe the flow behavior of the granular material and additionally considers the effect of segregation.

This can be done using the discrete element method (DEM). But due to the long computation times of the DEM for large particle systems, a macroscopic model is a natural choice to simulate large industrial processes. Such continuum models already have been found to describe the flow behavior of granular materials, like the hydrodynamic model of Latz and Schmidt [1], which we will present and modify in the next section. Unfortunately, all these models need to be fitted to experimental data and are not able to additionally describe the segregation effect. Also there are promising continuum models that describe the effect

\footnotetext{
^e-mail: dominik.gilberg@itwm.fraunhofer.de
}

of size-segregation. One of the first models of this kind was proposed by Bridgwater, Foo and Stephens in 1985 [2]. Later on models of similar structure were derived by Savage and Lun [3] or Gray and Thornton [4]. Larcher and Jenkins [5] extended kinetic theory for binary mixtures of nearly elastic spheres to predict the evolution of the concentration fractions of the two species. The modeling steps performed by Gray and Thornton are the basis of the segregation model we present in the upcoming section. In the last years extensions to their model were derived including size and density segregation [6],[7], but they are all modeled for given flow fields of idealized avalanches.

In the next section we present the mathematical models for the flow behavior and the segregation. We combine them to derive a model that shows the segregation effect in quite arbitrary flow fields. In Section 3 we present first simulation results gained by the model.

\section{The mathematical models}

The mathematical model consists of a hydrodynamic model of Navier-Stokes-Type describing the behavior of granular flows, and of a segregation equation, based on mixture theory. It is assumed that the granular mixture consists of small and large particles with equal material densities $\rho^{s *}=\rho^{l *}=$ const. In mixture theory one assumes that each constituent $v$ is present everywhere. Its percentage of the local volume is given by the volume fraction $\phi^{v}$. 
Hence, in every point it holds

$$
\phi^{s}+\phi^{l}+\phi^{a}=1,
$$

where $\phi^{s}, \phi^{l}$ is the volume fraction of small and large particles, respectively. The interstitial pore space filled with air is denoted by $\phi^{a}$. Further, the volume fraction of the whole granular material is given by

$$
\Phi=\phi^{s}+\phi^{l} \text {. }
$$

For the upcoming modeling process we do not ignore the volume occupied by air, as particles are not able to fill the whole space due to their shape. Nevertheless, we assume that the air has a passive role, where the pressure, and so the interaction on the particles is negligible small.

Classically, equations are formulated in terms of partial densities. They are given by a linear volume fraction scaling

$$
\rho^{v}=\phi^{v} \rho^{v *}
$$

that equally holds for the granular mixture

$$
\rho=\Phi \rho^{*} .
$$

To avoid further variable changes all equations are directly given in terms of the respective volume fractions, as all upcoming terms are linear in the density, and $\rho^{*}=\rho^{s *}=$ $\rho^{l *}=$ const.

The final model includes solely an equation for the behavior of the small particles as the large particles are given through the saturation condition (2). To simplify the notation in the final model, we write $\phi$ instead of $\phi^{s}$.

\subsection{The granular flow model}

The origin of the granular flow model is a set of hydrodynamic equations derived by Latz and Schmidt [1]. To reproduce the dilute flow regimes the model is based on kinetic theory. As granular materials come to rest they behave differently than in the fast dilute flow regimes. Kinetic theory is not able to reproduce this behavior correctly any more. Therefore, they modified the model using a theory from soil mechanics to extend its applicability to arresting granular flows. Their final set of hydrodynamic equations is a hybrid model of kinetic theory and the soil mechanical approach. We use a similar model for the granular flow as derived in [1]. As the original model does not take into account particles of different size, we apply some further modifications.

The granular system is given by the mass balance (6) and the momentum balance (7), that we solve for the granular volume fraction $\Phi$ and the mean granular velocity $\mathbf{u}$. One should keep in mind that the granular volume fraction $\Phi$ is bounded by a maximum volume fraction

$$
0 \leq \Phi \leq \Phi^{\max }(\phi)<1,
$$

due to the interstitial pore space between the particles. For particles of equal size, as in the original model, $\Phi_{\max }$ is a constant. But for a system of small and large particles it is a function that depends on the local amount of small and large particles.

In addition to equation (6) and (7) we solve equation (8) for the granular temperature $T$, that accounts for the mean fluctuating kinetic energy in the system.

$$
\begin{aligned}
\partial_{t} \Phi+\nabla \cdot(\Phi \mathbf{u}) & =0 \\
\partial_{t}(\Phi \mathbf{u})+\nabla \cdot(\Phi \mathbf{u} \otimes \mathbf{u}) & =\nabla \cdot \overline{\boldsymbol{\sigma}}+\Phi \mathbf{g}, \\
\partial_{t}(\Phi T)+\nabla \cdot(\Phi T \mathbf{u}) & =\frac{3}{2}(\eta \kappa: \kappa-\nabla \cdot \mathbf{q})-\varepsilon \Phi T
\end{aligned}
$$

The stress tensor in equation (7) is given by

$$
\overline{\boldsymbol{\sigma}}=\eta \kappa-p \mathbf{I}, \quad \kappa=\nabla \mathbf{u},
$$

where $\eta$ defines the granular viscosity and $p$ the pressure. The gravitational acceleration is denoted by $\mathbf{g}$. The temperature equation (8) is similar to a heat equation. The Term $\eta \kappa: \kappa$ is called viscous heating and acts as a source term for the granular temperature. Whereas, the last term on the right hand side is a sink, which accounts for the loss of energy due to inelastic collisions. The parameter $\varepsilon$ is the energy dissipation rate. If there are no forces acting on the granular material, a moving system will rapidly come to rest due to this term. The granular heat flux is given by

$$
\mathbf{q}=-\lambda \nabla T
$$

where $\lambda$ denotes the temperature viscosity.

Equations of state can be derived from kinetic theory to close the system [8]. The kinetic expressions are denoted by the subscript $k$. Additionally, so-called yield expressions are derived, that are solely active in the dense flow regime $\Phi>\Phi_{0}$. Accordingly, we use the subscript $y$. In the resulting hybrid model the pressure $p$ can be split into a kinetic and a yield part

$$
p=p_{k}+p_{y}
$$

with

$$
\begin{aligned}
& p_{k}=\Phi T g(\Phi, \phi) \\
& p_{y}=\Theta\left(\Phi-\Phi_{0}\right) T_{0}\left(\Phi-\Phi_{0}\right) g(\Phi, \phi),
\end{aligned}
$$

where $\Theta(\cdot)$ is the Heaviside step function and

$$
g(\Phi, \phi):=\left(1-\frac{\Phi}{\Phi^{\max }(\phi)}\right)^{-1}
$$

the compressibility factor. If the local amount of granular material is in a physically valid state $\Phi<\Phi^{\max }(\phi)$, the compressibility factor helps to ensure that the solution of the system stays valid. Similar to the pressure all transport coefficients in the system have a kinetic and an yield expression.

$$
\begin{array}{lll}
\eta=\eta_{k}+\eta_{y}, & \eta_{k}=\eta_{0} \sqrt{T} \Phi g(\Phi, \phi), & \eta_{y}=\eta_{k} \frac{p_{y}}{p_{k}} \\
\varepsilon=\varepsilon_{k}+\varepsilon_{y}, & \varepsilon_{k}=\varepsilon_{0} \sqrt{T} g(\Phi, \phi), & \varepsilon_{y}=\varepsilon_{k} \frac{p_{y}}{p_{k}} \\
\lambda=\lambda_{k}+\lambda_{y}, & \lambda_{k}=\lambda_{0} \sqrt{T} \Phi g(\Phi, \phi), & \lambda_{y}=\lambda_{k} \frac{p_{y}}{p_{k}} .
\end{array}
$$


In the dilute regime the yield pressure $p_{Y}$ is zero and so the yield part of each transport coefficient is zero, too. In this case all coefficients reduce to their kinetic expression. In the dense flow regime $p_{Y}$ is non zero. All parameters subscribed with 0 are constants, that either have to be looked up in literature or validated by experiments. A more detailed derivation of the model is given in [1].

\subsection{The segregation equation}

We assume that each constituent of the mixture can be described as a continuum. Additionally, we exclude agglomeration effects or breakage of particles. Therefore, they must fulfill the following mass and momentum balances:

$$
\begin{aligned}
\partial_{t} \phi^{v}+\nabla \cdot\left(\phi^{v} \mathbf{u}^{v}\right) & =0 \\
\partial_{t}\left(\phi^{v} \mathbf{u}^{v}\right)+\nabla \cdot\left(\phi^{v} \mathbf{u}^{v} \otimes \mathbf{u}^{v}\right) & =\nabla \cdot \overline{\boldsymbol{\sigma}}^{v}+\phi^{v} \mathbf{g}+\boldsymbol{\beta}^{v} .
\end{aligned}
$$

The interaction term $\boldsymbol{\beta}^{v}$ in equation (19) describes the force on particle phase $v$ due to the other particles.

To derive an equation for particle phase $v$, we start from the mass balance (18). Introducing the relative velocity of constituent $v$ to the bulk

$$
\mathbf{u}^{v m}=\mathbf{u}^{v}-\mathbf{u}
$$

we obtain the following form for the mass balance:

$$
\partial_{t} \phi^{v}+\nabla \cdot\left(\phi^{v} \mathbf{u}\right)=-\nabla \cdot\left(\phi^{v} \mathbf{u}^{v m}\right) .
$$

The left hand side of equation (21) describes the transport with the bulk velocity $\mathbf{u}$, which is given by the granular flow model. The right hand side represents the segregation as it is a relative motion to the bulk.

Segregation happens in the direction of the gravitational force. In flowing granular materials shear forces open small voids in the granular system. The small particles are more likely to fall into these empty spaces and percolate through the granular matrix. As a consequence the large particles end up at the top of the system.

To find an expression for the relative velocity $\mathbf{u}^{v m}$ we follow the idea of Gray and Thornton [4] and introduce a pressure scaling

$$
p^{v}=f^{v} p \quad \forall v .
$$

While the small particles percolate through the granular system they carry less of the overburden pressure than the large ones. Hence, $f^{v}$ is the proportion of the load carried by phase $v$, that must fulfill

$$
f^{s}+f^{l}=1 .
$$

We choose the load functions in a similar way to [4]

$$
f^{v}=\frac{1}{\Phi}\left(\phi^{v} \pm \frac{B}{\Phi} \phi^{s} \phi^{l}\right)
$$

where $B$ is a non-dimensional constant. The equation with " + stands for large particles and with " - " for small particles. The scalings by $\Phi$ are necessary to fulfill (23) and ensure that the effective segregation velocity is independent of $\Phi$, as we do not work with volume fractions relative to the granular volume.
For the derivation of the relative velocity we look at the momentum balance (19), as it contains the information of pressure and particle interactions. The expression of the interaction force $\boldsymbol{\beta}^{v}$ must fulfill Newtons third law, which reduces to

$$
\boldsymbol{\beta}^{s}+\boldsymbol{\beta}^{l}=0,
$$

as the air phase has no influence on the granular system. The interaction force $\boldsymbol{\beta}^{v}$ can be written as

$$
\boldsymbol{\beta}^{v}=p \nabla f^{v}-\phi^{v} \frac{\mu}{\dot{\gamma}}(\underbrace{\mathbf{u}^{v}-\mathbf{u}}_{\mathbf{u}^{v m}}) \quad \forall v .
$$

The first term ensures that the segregation process is driven by intrinsic rather than partial pressure gradients [4]. The second term is a drag term of Darcy type as proposed in [9], where $\mu$ is the coefficient of inter particle drag. It is inversely proportional to the shear rate $\dot{\gamma}$. Consequently, the drag reduces for an increasing shear rate [6],[10].

The shear rate can be approximated by the granular temperature. In a steady state the equation for the granular temperature (8) can be written as

$$
\frac{3}{2} \eta \dot{\gamma}^{2}=\varepsilon \Phi T
$$

With the expressions (15) and (16) the shear rate is proportional to the square root of the granular temperature

$$
\dot{\gamma}=\sqrt{\frac{2}{3} \frac{\varepsilon_{0}}{\eta_{0}} T} .
$$

The expression for the relative velocity $\mathbf{u}^{v m}$ is a direct consequence of the previous assumptions. Neglecting the acceleration terms in the momentum equation (19), assuming that the stress is given by a lithostatic pressure field [4] and combining the previous relations (22), (23), (26) and (28), the relative velocity for the small particles is given by

$$
\mathbf{u}^{s m}=\mathbf{S}^{s l} \frac{\phi^{l}}{\Phi}
$$

The mean segregation velocity

$$
\mathbf{S}^{s l}=\underbrace{-\frac{B}{\mu} \sqrt{\frac{2}{3} \frac{\varepsilon_{0}}{\eta_{0}}}}_{\mathbf{S}_{0}^{s l}} \mathbf{g} \sqrt{T}
$$

is zero for all directions orthogonal to the gravitational force. Further, the relative velocity vanishes, when the system reaches a static state as the granular temperature decreases. The factor $\mathbf{S}_{0}^{s l}$ needs to be validated by experiments or DEM simulations. With the help of the saturation condition (2) the final segregation equation for the small particle phase is given by

$$
\partial_{t} \phi+\nabla \cdot(\phi \mathbf{u})=-\nabla \cdot\left(\mathbf{S}^{s l} \frac{\Phi-\phi}{\Phi} \phi\right)
$$

with the temperature and gravity dependent segregation velocity

$$
\mathbf{S}^{s l}=\mathbf{S}_{0}^{s l} \mathbf{g} \sqrt{T} .
$$

The final system is given by the equations (6), (7), (8) and (31). 


\section{Simulation results}

To demonstrate the behavior of the combined model, we simulate a plain rotating tumbler filled to a height of $75 \%$ with glass beads of different sizes with radius ratio $\frac{r_{l}}{r_{s}}=2$. The material parameters for the glass beads are given in [11]. For the segregation strength, we choose $S_{0}^{s l}=1.5$, which is the same order as used in [4]. The setting is sensible as the processes in such a tumbler are already well known for two particle mixtures. There are flow regimes including segregation effects as well as static regimes. For the performed simulation the tumbler rotates in a clockwise direction with a speed of 0.05 rounds per second $(r p s)$. Initially, the system is perfectly mixed with equal volume fractions for small and large particles. For this setup a special pattern should form due to the rotation. Near the surface the particles are in motion. Except of the surface layers the granular material is in a static state. In the shearing top layers the segregation happens. Here the small particles segregate, but they do not even reach the core of the granular material, where the system is static already. In the static regime the material rotates with the tumbler without further segregation. Finally, a ring of small particles forms around a completely unchanged core. The large particles accumulate next to the walls [12].

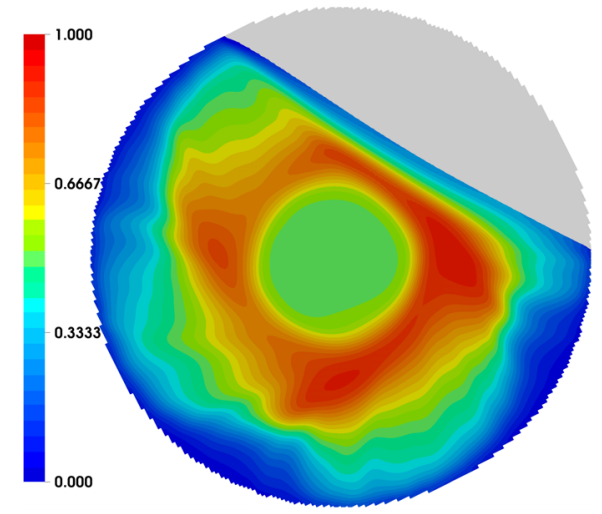

Figure 1. Volume fraction of the small particles relative to granular volume. The rotational velocity is $0.05 \mathrm{rps}$. The color table is fixed between 0 (blue) and 1 (red).

Figure 1 shows the cross-section of the tumbler. The volume fraction of small particles relative to the granular volume is plotted after 30 seconds of rotation. One can see the expected pattern, where the small particles build a ringlike formation. The effect of the segregation process to the granular system can be seen in Figure 2. It shows the same state as Figure 1 for the granular volume fraction $\Phi$ in a rage of 0.56 and 0.66 . In a mono-disperse system the packing profile is lithostatic. In a real application the maximum packing fraction for glass beads reaches a value of 0.632 . For our poly-disperse system this value can grow up to 0.68 , in regions with enough pressure and for $\phi^{s} \approx 0.3$. Our result shows the lithostatic profile superimposed by the segregation pattern. In the region of mainly one particle type the volume fraction fits to the values in mono-disperse system, whereas in the mixed regions the maximum packing fraction is bigger than 0.632 .

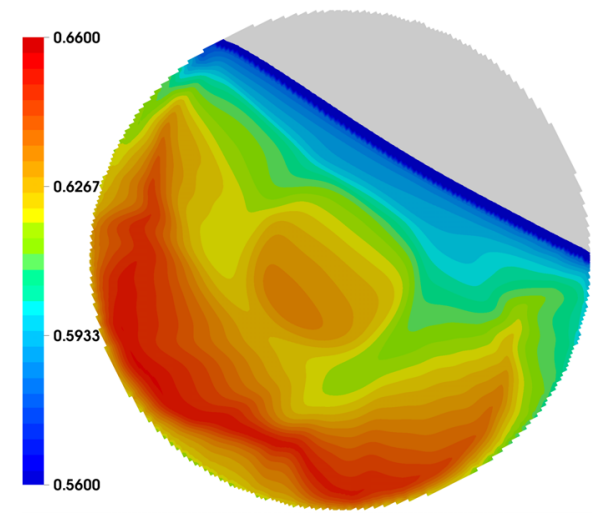

Figure 2. Volume fraction of the granular material. The rotational velocity is 0.05 rps. The color table is fixed between 0.6 (blue) and 0.66 (red).

\section{Conclusion}

We modified and combined two models that are able to reproduce the radial segregation effect occurring in a rotating tumbler. The granular temperature dependence in the segregation equation regulates the segregation in the different regimes. Even if the simulation qualitatively leads to good results, one should keep in mind, that the applicability of the model to other processes still needs to be tested and validated by experiments or DEM simulations.

\section{References}

[1] A. Latz, S. Schmidt, Granular Matter 12, 387 (2010)

[2] J. Bridgwater, W.S. Foo, D.J. Stephens, Powder Technology 41, 147 (1985)

[3] S.B. Savage, C.K.K. Lun, Journal of Fluid Mechanics 189, 311 (1988)

[4] Gray, J. M. N. T., A.R. Thornton, Proceedings of the Royal Society A: Mathematical, Physical and Engineering Sciences 461, 1447 (2005)

[5] M. Larcher, J. Jenkins, Journal of Fluid Mechanics 782, 405 (2015)

[6] D.R. Tunuguntla, O. Bokhove, A.R. Thornton, Journal of Fluid Mechanics 749, 99 (2014)

[7] Gray, J. M. N. T., C. Ancey, Journal of Fluid Mechanics 779, 622 (2015)

[8] N. Brilliantov, T. Pöschel, Kinetic theory of granular gases, Oxford graduate texts (Oxford University Press, Oxford, 2004), ISBN 0198530382

[9] L.W. Morland, Surv. Geophys. (Surveys in Geophysics) 13, 209 (1992)

[10] B. Marks, P. Rognon, I. Einav, Journal of Fluid Mechanics 690, 499 (2012)

[11] D. Niedziela, S. Rau, K. Steiner, S.d. Vita, M. Lutsche, M. Richter, M. Schmidt, To appear in: Chemical Engineering \& Technology (2017)

[12] I.S. Aranson, L.S. Tsimring, Reviews of Modern Physics 78, 641 (2006) 